\title{
Quantification of energy losses in organic solar cells from temperature-dependent device characteristics
}

\author{
Ulrich Hörmann, ${ }^{1}$ Julia Kraus, ${ }^{1}$ Mark Gruber, ${ }^{1}$ Christoph Schuhmair, ${ }^{1}$ Theresa Linderl, ${ }^{1}$ Stefan Grob, ${ }^{1}$ Stephan Kapfinger, ${ }^{1,2}$ \\ Konrad Klein, ${ }^{3}$ Martin Stutzman, ${ }^{2,3}$ Hubert J. Krenner, ${ }^{1,2}$ and Wolfgang Brütting ${ }^{1, *}$ \\ ${ }^{1}$ Institut für Physik, Universität Augsburg, Universitätsstrasse 1, 86159 Augsburg, Germany \\ ${ }^{2}$ Nanosystems Initiative Munich, Schellingstr. 4, 80799 München, Germany \\ ${ }^{3}$ Walter Schottky Institut, Technische Universität München, Am Coulombwall 4, 85748 Garching, Germany
}

(Received 8 October 2013; published 11 December 2013)

\begin{abstract}
Owing to the excitonic nature of photoexcitations in organic semiconductors, the working mechanism of organic solar cells relies on the donor-acceptor (D/A) concept enabling photoinduced charge transfer at the interface between two organic materials with suitable energy-level alignment. However, the introduction of such a heterojunction is accompanied by additional energy losses compared to an inorganic homojunction cell due to the presence of a charge-transfer (CT) state at the D/A interface. By careful examination of planar heterojunctions of the molecular semiconductors diindenoperylene (DIP) and $\mathrm{C}_{60}$ we demonstrate that three different analysis techniques of the temperature dependence of solar-cell characteristics yield reliable values for the effective photovoltaic energy gap at the D/A interface. The retrieved energies are shown to be consistent with direct spectroscopic measurements and the D/A energy-level offset determined by photoemission spectroscopy. Furthermore, we verify the widespread assumption that the activation energy of the dark saturation current $\Delta E$ and the CT energy $E_{\mathrm{CT}}$ may be regarded as identical. The temperature-dependent analysis of open-circuit voltage $V_{\mathrm{OC}}$ and dark saturation current is then applied to a variety of molecular planar heterojunctions. The congruency of $\Delta E$ and $E_{\mathrm{CT}}$ is again found for all material systems with the exception of copper phthalocyanine/ $\mathrm{C}_{60}$. The general rule of thumb for organic semiconductor heterojunctions, that $V_{\mathrm{OC}}$ at room temperature is roughly half a volt below the CT energy, is traced back to comparable intermolecular electronic coupling in all investigated systems.
\end{abstract}

DOI: 10.1103/PhysRevB.88.235307

PACS number(s): 73.40.Lq, 73.50.Pz, 73.61.Ph

\section{INTRODUCTION}

Organic solar cells (OSCs) have recently surmounted the important barrier of $10 \%$ efficiency for the conversion of incoming solar to electrical power, ${ }^{1}$ and the current record value for the power-conversion efficiency of molecular OSCs has even reached $12 \% .^{2}$ The rapid progress over recent years poses the question of where the fundamental efficiency limits of these materials are. ${ }^{3}$

For inorganic photovoltaics, this question was answered more than 50 years ago by the well-known Shockley-Queisser (SQ) limit. ${ }^{4}$ Based on very few fundamental assumptions, the SQ theory is able to predict an upper limit for the power-conversion efficiency of single-junction cells with the energy gap $E_{\mathrm{g}}$ of the active semiconductor material as the only input parameter. Owing to their excitonic nature, however, an important modification of this concept is required for OSCs, where a donor-acceptor heterojunction is used to enable charge separation. ${ }^{5}$ This is related to the existence of a chargetransfer (CT) state [see Fig. 1(a)] as an intermediate in the dissociation process from the photoexcited (excitonic) state on one molecular species towards free charge-separated states on both molecular species. ${ }^{6-9}$ The inclusion of the energy $E_{\mathrm{CT}}$ and absorption strength $\alpha_{\mathrm{CT}}$ of the CT state at the donor-acceptor interface in thermodynamic efficiency limits for OSCs has recently been demonstrated by different authors. ${ }^{10-13}$ They found that the photocurrent is not severely affected by the existence of a CT state, as long as its absorption strength is weak compared to that of the main absorber material. In that case the number of generated charge carriers remains governed by the fundamental absorption across the optical gap of the absorber. The open-circuit voltage $V_{\mathrm{OC}}$, on the other hand, is a measure of the chemical potential of the photogenerated charge-carrier pairs. As such, it is strongly affected by the introduction of the CT energy, which needs to be lower than the optical gap in order to enable charge transfer. Because of this fact $E_{\mathrm{CT}}$ takes the role of $E_{\mathrm{g}}$ in the derivation of the thermodynamic efficiency limits of OSCs and is sometimes also termed photovoltaic gap ${ }^{14}$ or effective gap. ${ }^{15}$ As a consequence, calculations predict both lower power-conversion efficiencies (20\% to $25 \%)$ compared to the classical SQ model and larger values for the optical gap, where the highest efficiency is achieved. ${ }^{11,13}$ Note that practical efficiency limits are lower by a factor of 2 if more realistic values ( $75 \%$ each) are chosen instead of $100 \%$ quantum efficiency and ideal fill factors. ${ }^{3,6,12,16,17}$

In the meantime the concept of an interfacial CT state is well established in polymer-fullerene bulk-heterojunction solar cells. Its existence has unambiguously been proven by spectral subgap absorption and photocurrent measurements as well as by electroluminescence spectra, ${ }^{18,19}$ and a consistent description of temperature-dependent device characteristics was obtained. ${ }^{20}$ These investigations could directly show that $E_{\mathrm{CT}}$ sets the upper limit for $V_{\mathrm{OC}}$ as temperature approaches $0 \mathrm{~K}$. However, for molecular semiconductors such a conclusive picture of the role of CT states at mostly planar donor-acceptor (D/A) heterojunctions is still missing. Rand et al. have presented the first systematic study of the interrelation between the measured open-circuit voltage and the energy-level offset $E_{\mathrm{DA}}$ on a range of D/A systems. ${ }^{21}$ They observed an increase of the open-circuit voltage $V_{\mathrm{OC}}$ with decreasing temperature until a saturation was reached. This maximum $V_{\mathrm{OC}}$ was found to correlate with the energy-level offset at the D/A interface, as estimated from the ionization potentials of the donor and 
(a)

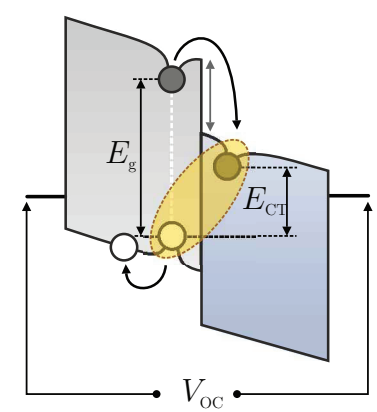

(b)

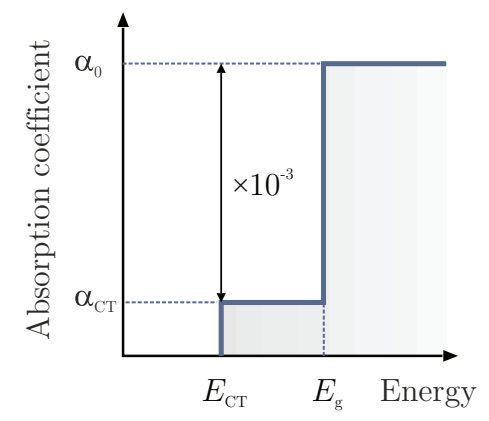

FIG. 1. (Color online) (a) Schematic illustration of the formation of a CT state with energy $E_{\mathrm{CT}}$. (b) Steplike absorption in a D/A heterojunction solar cell. In addition to the absorption $\alpha_{0}$ of photons with energy exceeding the optical energy gap $E_{\mathrm{g}}$, absorption from the CT state $\alpha_{\mathrm{CT}}$ already takes place for photon energies above $E_{\mathrm{CT}}$ but is typically several orders of magnitude weaker.

acceptor and the optical gap of the absorber. Potscavage et al. used the thermally activated behavior of the dark saturation current of solar cells to identify the crucial role of the energy-level offset at the D/A interface for $V_{\mathrm{OC}}{ }^{22}$ Perez et al. noted that in addition to the activation energy $\Delta E$ of the dark current under reverse-bias conditions, its prefactor, depending on the electronic coupling between both molecular species, is also important for the magnitude of the recombination current and thus determines $V_{\mathrm{OC}}{ }^{23}$ Giebink et al. have put forward an extended diode model for organic heterojunctions based on polaron pair generation and recombination processes. ${ }^{24}$ Remarkably, they predict two different ideality factors related to trapping in both materials forming the interface and apply their model to analyze current-voltage characteristics in terms of polaron pair recombination. However, in spite of the successful description of the temperature-dependent device behavior in the dark and under illumination, no direct spectroscopic evidence for the existence of a CT state at the respective energies was given. Note that the claimed evidence for an exciplex emission of $\mathrm{CuPc} / \mathrm{C}_{60}$ in Ref. 24 has to be considered as indirect evidence because no clear spectroscopic signature was found. Furthermore, the two different ideality factors postulated are expected to cause two different slopes in $V_{\mathrm{OC}}(T)$, which have not actually been observed. ${ }^{24}$

The aims of this paper are therefore, on the one hand, to establish temperature-dependent current-voltage characteristics (in the dark and under illumination) of planar heterojunction OSCs as a straightforward method for determining the effective photovoltaic gap and, on the other hand, to validate these results by comparison with photoelectron spectroscopy, optical absorption, and electroluminescence spectra. In particular, we will demonstrate that the different quantities $E_{\mathrm{CT}}, E_{\mathrm{DA}}$, and $\Delta E$ used in the literature are, within an experimental error of $\pm 0.1 \mathrm{eV}$, identical. For this purpose we will use the aromatic hydrocarbon diindenoperylene (DIP; $\mathrm{C}_{32} \mathrm{H}_{16}$ ) as the donor in combination with the fullerene $\mathrm{C}_{60}$ as the electron acceptor in planar heterojunction solar cells, where a broad range of experimental data has been gathered since this system was first introduced by Wagner et al. ${ }^{25}$ Thereafter the developed analysis will be applied to a broader range of molecular materials, including sexithiophene (6T), pentacene (Pen), and $\mathrm{Cu}$ phthalocyanine $(\mathrm{CuPc})$ as donors together with $\mathrm{C}_{60}$ and DIP as acceptors, to obtain a more general picture of energy losses in molecular OSCs.

\section{THEORETICAL CONCEPTS AND STATE OF THE ART}

\section{A. Dark-current activation energy}

In a simplistic picture the electrical behavior of a solar cell can be described as a current source in parallel with a diode, representing the voltage response of the device in the dark. These two contributions are added and yield the current density vs voltage $(j-V)$ characteristics: ${ }^{26,27}$

$$
j=j_{\text {dark }}-j_{\text {photo }}=j_{0}\left[\exp \left(q V / n k_{\mathrm{B}} T\right)-1\right]-j_{\mathrm{SC}},
$$

where the expression for $j_{\text {dark }}$ is also known as the Shockley equation. $j_{\text {SC }}$ is the short-circuit current density of the solar cell under illumination and is assumed to be identical to the photocurrent $j_{\text {photo }} \cdot j_{0}$ is the reverse saturation current density, $q$ is the elementary charge, $n$ is the diode ideality factor, $k_{\mathrm{B}}$ is Boltzmann's constant, and $T$ is the absolute temperature.

The saturation current density $j_{0}$, which sets the magnitude of the current in the absence of illumination, is also dependent on temperature according to ${ }^{26}$

$$
j_{0}=j_{00} \exp \left(-\Delta E / n k_{\mathrm{B}} T\right),
$$

where $j_{00}$ depends on the material system and is a measure for the coupling between the electronic states at the heterojunction. $\Delta E$ is the energy barrier at the interface, which has to be surmounted to generate a pair of oppositely charged carriers. In a classical $p$ - $n$ homojunction diode this would be the band gap of the semiconductor, while in a heterojunction device it denotes the band offset at the interface between the two different semiconductors. ${ }^{28}$ Accordingly, in organic heterojunctions this quantity can be identified with the energy offset between the highest occupied molecular orbital (HOMO) of the donor, where holes flow, and the lowest unoccupied molecular orbital (LUMO) of the acceptor, which is the relevant transport level for electrons. In inorganic $p-n$ junctions the ideality factor $n$, appearing in both equations, takes a value of 1 if direct recombination in the bulk dominates and 2 if trap-assisted recombination in the junction region is dominant. ${ }^{26}$ Note that Potscavage et al. have introduced two different ideality factors in both expressions, however, at the same time noting that they are virtually identical in actual devices. ${ }^{22}$ According to the Shockley equation, an analysis of temperature-dependent $j-V$ characteristics in the dark should thus allow for a determination of $\Delta E$ as the activation energy of $j_{0}$. This analysis has been applied by various authors, ${ }^{21-24,29}$ and the outcome was compared to the energy-level difference $E_{\mathrm{DA}}$ at the D/A interface predicted from photoelectron spectroscopy or cyclic voltammetry. Note that some authors used a modification of Eq. (2) with an additional factor of 2 in the denominator ${ }^{21,23}$ to obtain a better agreement with expected values for $E_{\mathrm{DA}}$; however, as we will briefly address later on, this seems to be a peculiarity of the investigated systems and not generic.

If the cell is illuminated (typically under simulated sunlight conditions) without an external load, there will be no net current $\left(j_{\text {dark }}=j_{\text {photo }}\right)$, and the voltage drop between both 
contacts equals the open-circuit voltage $V_{\mathrm{OC}}$, which is therefore given by

$$
q V_{\mathrm{OC}}=n k_{\mathrm{B}} T \ln \left(j_{\mathrm{SC}} / j_{0}-1\right) .
$$

Inserting Eq. (2) yields

$$
q V_{\mathrm{OC}} \approx \Delta E-n k_{\mathrm{B}} T \ln \left(j_{00} / j_{\mathrm{SC}}\right),
$$

where the assumption that $\exp \left(q V_{\mathrm{OC}} / n k_{\mathrm{BT}}\right) \gg 1$ has been used. This identifies $\Delta E / q$ as the limit of $V_{\mathrm{OC}}$ for $T \rightarrow 0$.

\section{B. The detailed balance approach}

Following the approach taken by Shockley and Queisser, i.e., a detailed balance limit, the photocurrent of the cell $j_{\mathrm{SC}}$ and the dark saturation current $j_{0}$ can be expressed by integrals over the radiation absorbed and emitted by the cell, respectively. The absorbed light is given by the solar spectrum (at the surface of the earth) $\Phi_{\text {sun }}(E)$, and the emitted radiation is given by a blackbody spectrum at $300 \mathrm{~K} \Phi_{\text {cell }}(E)$, both weighted by the absorption coefficient $\alpha(E)$ of the cell. ${ }^{4}$ This yields

$$
\begin{gathered}
j_{\mathrm{SC}}=q \int_{0}^{\infty} \alpha(E) \Phi_{\mathrm{sun}}(E) d E, \\
j_{0}=q \int_{0}^{\infty} \alpha(E) \Phi_{\text {cell }}(E) d E .
\end{gathered}
$$

Therein, the absorption spectrum $\alpha(E)$, originally resembling a step function with $\alpha(E)=0$ for $E<E_{\mathrm{g}}$ and $\alpha(E)=1$ for $E>E_{\mathrm{g}}$, needs to be modified to account for the presence of a CT state with energy $E_{\mathrm{CT}}$ at the D/A interface in organic solar cells. For example, a double-step function is commonly used [see Fig. 1(b)]: ${ }^{11-13}$

$$
\alpha(E)=\left\{\begin{aligned}
0, & E<E_{\mathrm{CT}}, \\
\alpha_{\mathrm{CT}}, & E_{\mathrm{CT}}<E<E_{\mathrm{g}}, \\
\alpha_{0}, & E>E_{\mathrm{g}},
\end{aligned}\right.
$$

where $\alpha_{\mathrm{CT}}$ stands for the absorption strength of the CT state (relative to the fundamental absorption across the gap being set to unity). Following this approach, $V_{\mathrm{OC}}$ is readily calculated from Eq. (3) by the postulate that under open-circuit conditions all photogenerated carriers have to recombine so that no net current flows in the external circuit. Numerical calculations (for details see Ref. 13) predict an almost-linear increase in $V_{\mathrm{OC}}$ with decreasing temperature, leading to $E_{\mathrm{CT}} / q$ as the upper limit at $T=0$.

Note that Eq. (4) also predicts a linear temperature dependence of $V_{\mathrm{OC}}$ with $\Delta E$ as the limiting value for $T \rightarrow 0$. However, in the following we will identify the extrapolated upper limit of $V_{\mathrm{OC}}$ with the energy $E_{\mathrm{CT}}$ of the interfacial CT state, where recombination of charge carriers occurs. We do not $a$ priori set this value equal to $\Delta E$, which is relevant for carrier generation across the heterojunction barrier in the reverse-bias direction.

In contrast to the idealized situation in the original SQ model, where all recombination was assumed to be radiative, the occurrence of predominantly nonradiative recombination in real cells can be taken into account by an enhanced recombination current $j_{0}^{\text {(nonrad) }}=k_{n} j_{0}^{\text {(rad) }}{ }^{13}$ Rau et al. have shown that $k_{n}$ is equal to the inverse of the electroluminescence quantum efficiency of a solar cell. ${ }^{30}$ Performing this procedure for varying temperature, it follows again that $q V_{\mathrm{OC}}(T \rightarrow 0)=$ $E_{\mathrm{CT}}$; that is, the upper limit of the open-circuit voltage remains unchanged. ${ }^{13}$ Furthermore, knowing the relative strength of nonradiative recombination, it is now possible to express losses in $V_{\mathrm{OC}}$ at finite temperature as radiative and nonradiative ones:

$$
V_{\mathrm{OC}}(T)=E_{\mathrm{CT}} / q-\Delta V_{\mathrm{OC}}^{(\mathrm{rad})}-\Delta V_{\mathrm{OC}}^{(\mathrm{nonrad})} .
$$

Vandewal et al. have recently applied the detailed balance approach to polymer bulk-heterojunction solar cells. ${ }^{20}$ They have been able to relate $V_{\mathrm{OC}}$ and its losses to spectroscopically accessible quantities at the donor-acceptor interface. These are, in particular, the energy of the CT state $E_{\mathrm{CT}}$, the reorganization energy $\lambda_{0}$ in forming the CT state by light absorption, and a parameter $f$ that is proportional to the number of CT states in the device as well as the electronic coupling between CT and the ground state:

$$
\begin{aligned}
V_{\mathrm{OC}}(T)= & E_{\mathrm{CT}} / q+\left(k_{\mathrm{B}} T / q\right) \ln \left(\frac{j_{\mathrm{SC}} h^{3} c^{2}}{2 \pi f q\left(E_{\mathrm{CT}}-\lambda_{0}\right)}\right) \\
& +\left(k_{\mathrm{B}} T / q\right) \ln \left(\eta_{\mathrm{EQE}, \mathrm{EL}}\right),
\end{aligned}
$$

where $h$ and $c$ are Planck's constant and the speed of light, respectively, and $\eta_{\mathrm{EQE}, \mathrm{EL}}$ denotes the electroluminescence quantum efficiency. Using experimental parameters from photocurrent and electroluminescence spectra, the authors have been able to achieve a consistent description of the temperature dependence of $V_{\mathrm{OC}}$ for a range of polymerfullerene-blend solar cells. In particular, they could show that the open-circuit voltage measured at room temperature (under one sun illumination) is typically between 0.5 and $0.6 \mathrm{~V}$ less than the CT energy of the respective D/A interface. ${ }^{20}$

In the following we will use DIP/ $\mathrm{C}_{60}$ as a prototypical molecular donor-acceptor system to establish a conclusive model for the origin of the open-circuit voltage from the offset energy at the D/A interface. By a priori independent consideration of the energy values obtained by photoemission spectroscopy $\left(E_{\mathrm{DA}}\right)$, dark current analysis $(\Delta E)$, and the detailed balance approach $\left(E_{\mathrm{CT}}\right)$ as well as optical spectroscopy $\left(E_{\mathrm{CT}}^{\mathrm{opt}}\right)$, we show that these energies are related. We present various methods that consistently yield the effective photovoltaic energy gap at the heterojunction.

\section{MATERIALS AND METHODS}

Organic solar cells in planar heterojunction (PHJ) architecture [see Fig. 2(a)] were fabricated on commercially available indium tin oxide (ITO) coated glass substrates (purchased from Thin Film Devices, Inc., Anaheim, CA; sheet resistance $\approx 20 \Omega$ /square). Prior to the evaporation of the active D/A heterojunction, an approximately 30-nm-thick layer of poly(3,4-ethylenedioxythiophene):poly(styrenesulfonate) (PEDOT:PSS; purchased from Heraeus Clevios GmbH, Leverkusen, Germany) was spin coated from an aqueous solution and annealed at $125^{\circ} \mathrm{C}$ for 45 min on a hotplate under ambient conditions. Subsequently, the active donor and acceptor layers as well as a 5-nm layer of the exciton blocking material bathocuproine (BCP; purchased from Sigma Aldrich as sublimed grade and used without further purification) were deposited using the standard thermal evaporation procedure at base pressures of $10^{-6}-10^{-7}$ mbar. Finally, $100 \mathrm{~nm}$ of 
(a)

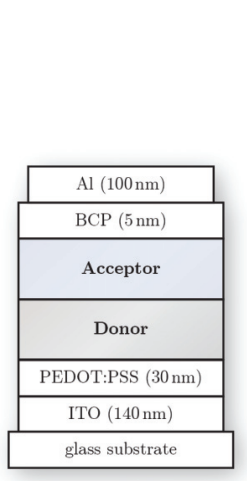

(b)

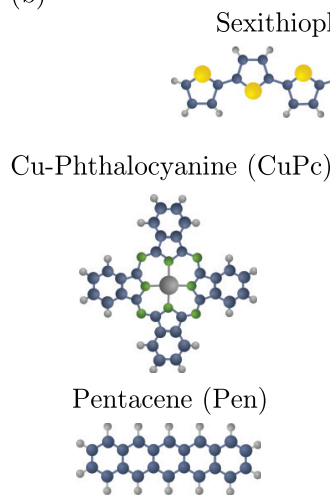

(c)

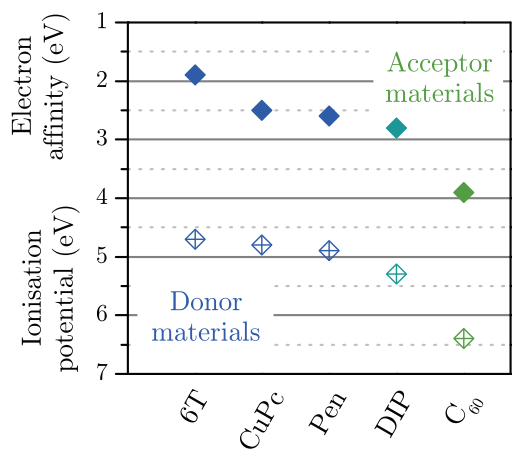

FIG. 2. (Color online) (a) Device stack of the investigated planar heterojunctions. (b) Chemical structures of the active organic semiconductors $\alpha$-sexithiophene (6T), copper phthalocyanine ( $\mathrm{CuPc}$ ), diindenoperylene (DIP), pentacene (Pen), and Buckminster fullerene $\left(\mathrm{C}_{60}\right)$. (c) Energies of the HOMO (open symbols) and LUMO (solid symbols) levels for the investigated materials. The values are taken from the literature. ${ }^{14,31,32}$ The LUMO levels of CuPc and Pen are estimated by adding transport gaps of $2.3 \mathrm{eV}$ (Ref. 33) and 2.2 eV (Ref. 34) to the respective HOMOs. The LUMO of $6 \mathrm{~T}$ is estimated from the optical gap of $6 \mathrm{~T}\left(E_{\mathrm{g}}=2.4 \mathrm{eV}\right)$ and the relation between the optical gap and the transport gap, both found in Ref. 35.

aluminum were evaporated through a shadow mask as a top electrode, resulting in solar cells with an active area of $A=4 \mathrm{~mm}^{2}$. The active materials, diindenoperylene (DIP; purchased from S. Hirschmann, Universität Stuttgart, Stuttgart, Germany), $\alpha$-sexithiophene (6T; purchased from Sigma Aldrich), and $\mathrm{C}_{60}$ (purchased from Creaphys, Dresden, Germany), were purified twice by gradient sublimation. Copper phthalocyanine $(\mathrm{CuPc})$ was purchased from Sigma Aldrich as sublimed grade and was additionally purified once by gradient sublimation. Pentacene (Pen; purchased from Sigma Aldrich) was used without further purification. The chemical structures of the organic semiconductors as well as the literature values of their transport levels are depicted in Figs. 2(b) and 2(c). For the PHJ solar cells, the exact film thicknesses of the active layers are specified in the results section. Please note that growth conditions and film thicknesses have not been optimized for all devices. All evaporated organic layers have been grown while the substrate was kept at room temperature. Only in the case of DIP donor films in solar cells was an elevated substrate temperature of $100^{\circ} \mathrm{C}$ used to avoid injection barriers. ${ }^{36}$

Current density vs voltage $(j-V)$ characteristics of the solar cells were recorded using a source measure unit (Keithley 236 SMU) in the dark and under illumination. Unless otherwise stated, a white light-emitting diode (LED; Luxeon LXHLNWE8) was used as the light source. Since the illumination conditions do not fulfill the AM $1.5 \mathrm{~g}$ standard, values for power-conversion efficiencies are not specified. However, representative data can be found in Ref. 25 .

Temperature-dependent $j-V$ characteristics were measured in an inert-gas atmosphere (300-mbar $\mathrm{N}_{2}$ ) using a liquidnitrogen-cooled cryostat (Cryovac) into which the samples were transferred without air exposure. Temperature was measured using a sensor mounted in close proximity to the sample. Temperature-dependent measurements of $V_{\mathrm{OC}}$ and $j_{\mathrm{SC}}$ have been recorded for different illumination intensities ranging from approximately 0.5 to $0.5 \times 10^{-3}$ sun.

Photothermal deflection spectroscopy (PDS) was applied to neat and mixed films (ratio of approximately 1:1) of DIP and
$\mathrm{C}_{60}$ (co)evaporated on quartz glass substrates. Perfluorohexane was used as the deflection medium, which assures high transmittance in the energy region of $0.6-6 \mathrm{eV}$ and large changes in index of refraction with small changes in temperature. Moreover, it dissolves neither DIP nor $\mathrm{C}_{60}$.

Electroluminescence (EL) measurements were carried out on cells with a planar-mixed heterojunction (PM-HJ) with a coevaporated active layer of DIP: $\mathrm{C}_{60}$ and compared to single-layer devices with neat DIP or $\mathrm{C}_{60}$. EL measurements were realized by using a liquid-nitrogen-cooled CCD camera (PyLoN:100BR, Princeton Instruments) with a spectral sensitivity in the wavelength range of approximately 300-1000 nm. All EL measurements were performed on encapsulated solar cells under a dc voltage drive from a Keithley source meter.

\section{EXPERIMENTAL RESULTS AND DISCUSSION}

\section{A. $\mathrm{DIP} / \mathrm{C}_{60}$ as a prototypical molecular donor-acceptor system}

\section{Temperature-dependent $j-V$ characteristics in the dark: Analysis of saturation current and ideality factor}

Earlier studies on the material combination DIP/ $\mathrm{C}_{60}$ have proven its suitability as a donor-acceptor pair in planar and mixed heterojunction solar cells. ${ }^{25,36}$ Concerning its application as a donor in organic solar cells, the main advantage of DIP can be found in its high ionization potential and the concomitant favorable energy-level alignment with the acceptor $\mathrm{C}_{60}$, leading to high open-circuit voltages exceeding $0.9 \mathrm{~V} .{ }^{25}$ Moreover, favorable film morphology together with high crystalline order allows for improved transport properties of both excitons ${ }^{37}$ and charges ${ }^{38}$ in photovoltaic cells, resulting in high fill factors up to $75 \% .{ }^{25}$ To study its temperature-dependent behavior, dark $j-V$ characteristics have been recorded in the temperature range from 350 to $130 \mathrm{~K}$ for a planar heterojunction cell consisting of ITO/PEDOT:PSS/DIP $(50 \mathrm{~nm}) / \mathrm{C}_{60}(80 \mathrm{~nm}) / \mathrm{BCP}(5 \mathrm{~nm}) / \mathrm{Al}$ [see open symbols in Fig. 3(a)]. Based on the general diode equation (1), the exponential part of the forward-bias 
(a)
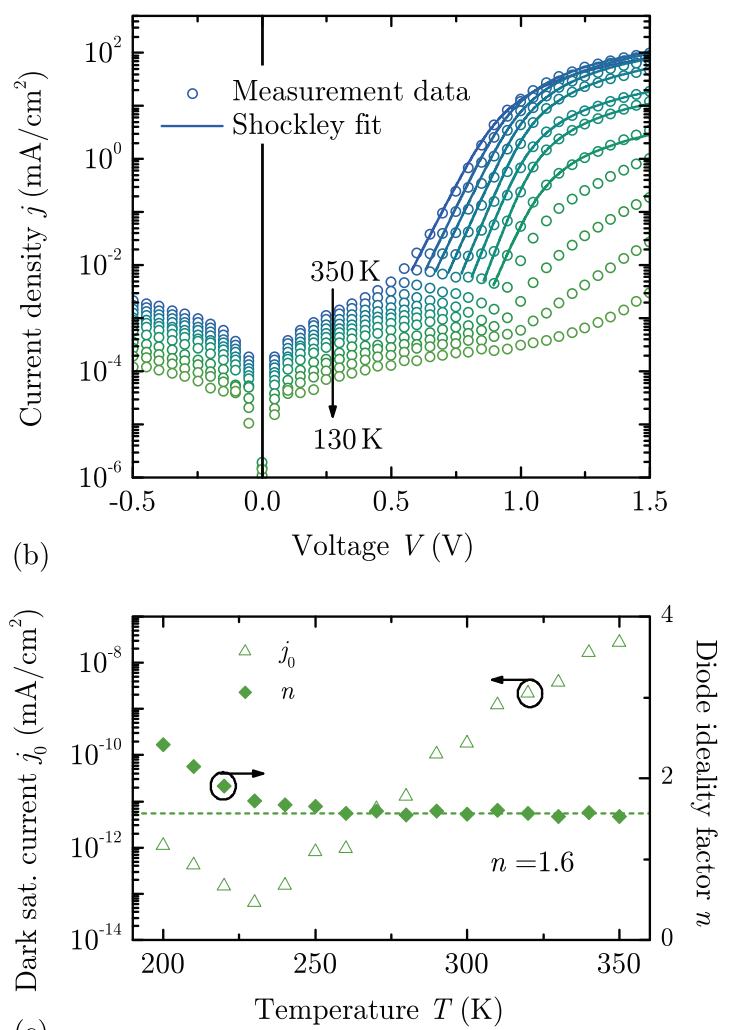

(c)

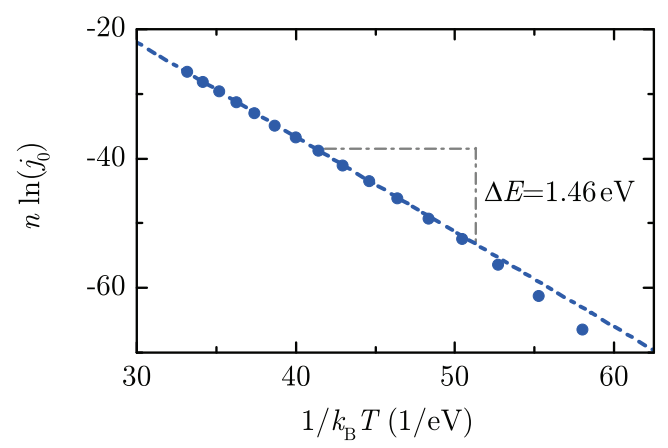

FIG. 3. (Color online) (a) Dark $j-V$ characteristics of a planar heterojunction with structure ITO/PEDOT:PSS/DIP(50 nm)/ $\mathrm{C}_{60}(80 \mathrm{~nm}) / \mathrm{BCP}(5 \mathrm{~nm}) / \mathrm{Al}$ recorded for several temperatures ranging from 130 to $350 \mathrm{~K}$ (open symbols). The solid lines are fitted curves in the forward-bias region using the Shockley equation. (b) Dark saturation current density $j_{0}$ and diode ideality factor $n$ depending on temperature, analyzed with the Shockley equation. (c) Temperature dependence of the dark saturation current evaluated by studying $n \ln \left(j_{0}\right)$ plotted versus $1 / k_{\mathrm{B}} T$ in order to estimate the activation energy $\Delta E$ from Eq. (2). For clarity only a subset of temperatures is shown in (a).

characteristics can be described using a modified Shockley equation:

$$
j(V)=j_{0}\left\{\exp \left[\frac{q\left[V-j(V) R_{\mathrm{S}} A\right]}{n k_{\mathrm{B}} T}\right]-1\right\},
$$

where an additional specific series resistance $R_{\mathrm{S}} A$ accounts for the slower increase of the current within the drift-dominated regime at high voltages. While the Shockley equation was originally applied for analyzing and parameterizing the
$j-V$ characteristics of inorganic $p-n$ junction solar cells, ${ }^{26}$ it was recently successfully adapted to organic solar cells, ${ }^{39,40}$ even in its modified variant. ${ }^{23}$ By a fit with the modified Shockley equation (10) $j_{0}$ and $n$ can be extracted from the data.

The obtained temperature dependencies of $n$ and $j_{0}$ are depicted in Fig. 3(b). Within the wide range of $240 \mathrm{~K} \leqslant T \leqslant$ $350 \mathrm{~K}$, the ideality factor shows an almost-constant value of $n \approx 1.6$ (dashed line), while the dark saturation current obeys a temperature dependence of the form $\ln \left(j_{0}\right) \propto 1 / k_{\mathrm{B}} T$. The deviation from this behavior below $240 \mathrm{~K}$ may be attributed to nonideal shunt and series resistances that become relevant at low temperatures. ${ }^{41}$ According to Eq. (2), an activation energy of $\Delta E=(1.46 \pm 0.03) \mathrm{eV}$ can be determined from plotting $n \ln \left(j_{0}\right)$ versus $1 / k_{\mathrm{B}} T$ [see Fig. 3(c)].

\section{Temperature-dependent $j-V$ characteristics under illumination: Analysis and extrapolation of open-circuit voltage}

The corresponding $j-V$ curves of the same PHJ DIP/C 60 solar cell under white LED illumination corresponding to roughly 0.2 sun are shown in Fig. 4(a). The short-circuit current density stays almost constant in the high-temperature range; however, when cooling below $T=180 \mathrm{~K}$, it markedly decreases, and the curves are characterized by pronounced $S$ shapes; that is, they show a decrease of the current close to $V_{\mathrm{OC}}$ and in the forward direction of the diode. Assuming a temperature-independent exciton generation rate, the reasons for the continuously decreasing $j_{\mathrm{SC}}$ can, on the one hand, be found in a reduction of charge-carrier extraction efficiency as a result of thermally activated charge-carrier mobility. On the other hand, a decline of exciton transport may contribute to the temperature dependence of $j_{\mathrm{SC}}$.

Regarding the temperature dependence of the open-circuit voltage, an almost-linear increase in $V_{\mathrm{OC}}$ with decreasing $T$ is observed in a wide temperature range [see Fig. 4(b)]. A similar relation has experimentally been found for a variety of material systems. ${ }^{21,42,43}$ The deviation from the linear behavior for temperatures $T<150 \mathrm{~K}$ can be attributed to the series resistance, which is found to strongly depend on temperature, as will be discussed below. Theoretical considerations predict this approximately linear temperature dependence of $V_{\mathrm{OC}}{ }^{13,44}$ This is based on the notion that the contribution of entropy to the electrochemical potential vanishes at $0 \mathrm{~K}$ (Ref. 45) and the energy gap that is responsible for charge recombination $\left(E_{\mathrm{CT}}\right)$ sets the upper limit for $q V_{\mathrm{OC}}$. Following this approach, an extrapolation of the linear behavior towards $T=0 \mathrm{~K}$ yields a value of $E_{\mathrm{CT}}=(1.42 \pm 0.02) \mathrm{eV}$.

Equation (4) shows that the dark saturation current has a decisive impact on the open-circuit voltage and presumably also on its temperature dependence. According to this relation, the open-circuit voltage can be calculated solely based on $\Delta E, n$, and $j_{00}$ extracted from dark $j-V$ characteristics, together with the short-circuit current density $j_{\mathrm{SC}}$ under given illumination conditions. As can be seen from Fig. 4(b) (blue diamonds), a very good agreement with the measured open-circuit voltage is obtained. The slight difference between the values determined for $\Delta E$ and $E_{\mathrm{CT}}$ becomes visible in the small deviation of reconstructed $V_{\mathrm{OC}}$ from measured $V_{\mathrm{OC}}$ data. 


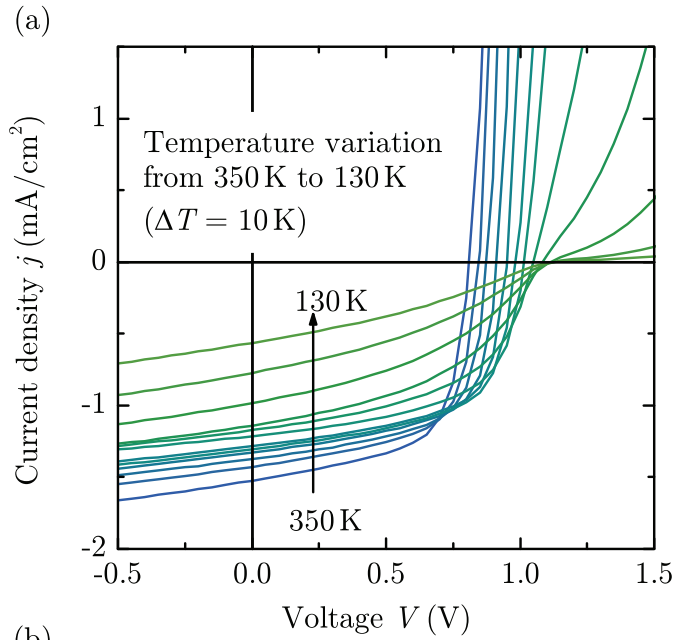

(b)

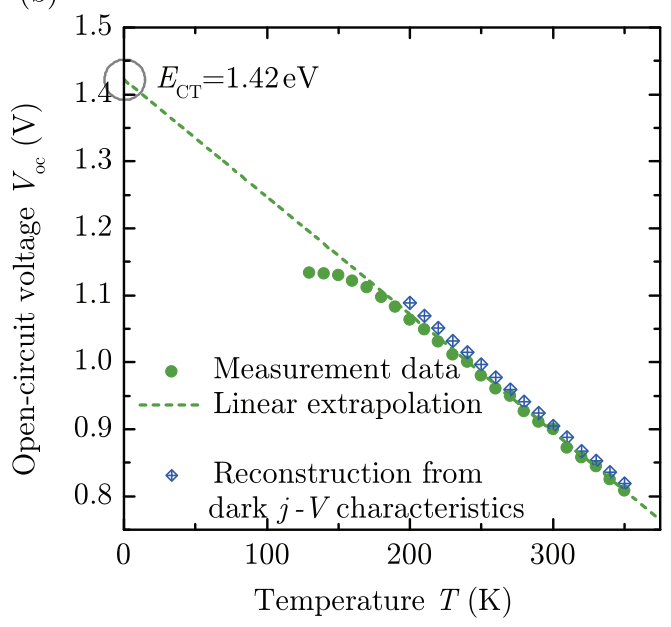

FIG. 4. (Color online) (a) $j$ - $V$ characteristics of a planar heterojunction solar cell under white LED illumination in the temperature range $130 \mathrm{~K} \leqslant T \leqslant 350 \mathrm{~K}, \Delta T=10 \mathrm{~K}$. The device has the following structure: ITO/PEDOT:PSS/DIP(50 nm)/C 60 ( $80 \mathrm{~nm}) / \mathrm{BCP}(5 \mathrm{~nm}) / \mathrm{Al}$. (b) Open-circuit voltage $V_{\mathrm{OC}}$ depending on temperature revealed from illuminated $j$ - $V$ data shown in (a) (green circles) with an extrapolation of the linear regime (dashed line) and reconstructed data according to Eq. (4) (diamond-shaped blue symbols). For clarity only a subset of temperatures is shown in (a).

Please note that the reconstruction has only been performed down to $200 \mathrm{~K}$, where $j_{\text {SC }}$ starts decreasing more substantially.

\section{Intensity and temperature-dependent open-circuit voltage: Analysis of saturation current and ideality factor}

Figure 5(a) shows the temperature dependence of the opencircuit voltage for different illumination intensities from 0.5 to $0.5 \times 10^{-3}$ sun using a solar simulator and neutral density filters, measured on a DIP/C 60 -based PHJ solar cell with a structure similar to that investigated before. The solid lines in Fig. 5(a) show the simulated temperature dependence for the different intensities. By solving Eqs. (6) and (3) and accounting for additional nonradiative recombination, the temperature dependence of $V_{\mathrm{OC}}$ can be calculated with a single value of $E_{\mathrm{CT}}=1.4 \mathrm{eV}$ for all intensities (for details refer to Ref. 13). However, the reduced photocurrent alone [as indicated by Eq. (3)] is insufficient to explain the steeper slope at low intensities. Significantly larger values of the dark saturation current $j_{0}$ have to be used. To account for this circumstance, a biexponential function relating the nonradiative recombination current to light intensity can be used (not shown here).

Just like the simulated curves, the extrapolation of the linear regimes for the various illumination intensities all coincide in the same value of $E_{\mathrm{CT}}=1.4 \mathrm{~V}$ at $T=0 \mathrm{~K}$, as expected from Eq. (4). In fact the simulated lines in Fig. 5(a) can hardly be distinguished from a simple linear extrapolation. This demonstrates that the determination of the CT energy by linear extrapolation of $V_{\mathrm{OC}}$ to $0 \mathrm{~K}$ is actually valid. However, this does not a priori mean that all curves can be described with a single set of parameters $\Delta E, n$, and $j_{00}$.

For a closer look into this issue, the measured $V_{\mathrm{OC}}(T)$ data sets are plotted vs $j_{\mathrm{SC}}$, which is approximately proportional to the illumination intensity if the slight decrease of $j_{\mathrm{SC}}$ with decreasing temperature [see Fig. 4(a)] is ignored. Figure 5(b) shows that, apart from the lowest two intensities, the proportionality of $V_{\mathrm{OC}}$ to $\log \left(j_{\mathrm{SC}}\right)$ according to Eq. (3) is well fulfilled. The fitted straight lines yield a temperature-dependent set of values for $n$ and $\ln \left(j_{0}\right)$ that are plotted versus $1 / k_{\mathrm{B}} T$, as shown in Fig. 5(c). The perfect linear relation confirms the validity of this approach and yields an activation energy of $\Delta E=(1.43 \pm 0.03) \mathrm{eV}$, in excellent agreement with the analysis of the dark saturation current shown in Fig. 3. For the lowest two light intensities $V_{\mathrm{OC}}$ falls below the straight lines in Fig. 5(b). This shows that $V_{\mathrm{OC}}$ is disproportionately reduced at low intensity, implying severely increased nonradiative recombination losses. This is in accordance with what has been observed by simulation and can be seen as an indication that trap-assisted recombination becomes dominant at low light intensity, leading to enhanced nonradiative recombination. ${ }^{36,46}$

\section{Direct spectroscopic evidence for the charge-transfer state from absorption and electroluminescence measurements}

While the electrical investigations discussed so far give more indirect information on the energetics at the D/A interface, direct spectroscopic evidence can be obtained from absorption and electroluminescence measurements. Note that for clear distinction the CT energy derived from spectroscopic measurements is denoted by $E_{\mathrm{CT}}^{\mathrm{opt}}$. As CT absorption features are typically characterized by low absorption coefficients, direct detection requires very sensitive spectroscopic techniques. Among the most common methods is PDS, which allows the determination of values for $\alpha_{\mathrm{CT}}$ that are several orders of magnitude smaller compared to the bulk material absorption. PDS is based on local heating of the sample which is caused by even very weak absorption of light. Figure 6(a) shows PDS data of a coevaporated mixed layer of DIP and $\mathrm{C}_{60}$ compared to the spectra of the single materials $(0.5-1 \mu \mathrm{m}$ on quartz). To obtain absolute values of the absorption coefficient, the PDS signal is calibrated using standard reflection/transmission measurements in the high-energy regime (open symbols), where both measurement ranges overlap. ${ }^{13}$

The DIP spectrum is characterized by a steep increase of absorption around $E=2.0 \mathrm{eV}$, presumably originating from tail states of the DOS distribution below the optical gap at about $2.25 \mathrm{eV}$. The tiny peak at $1.1 \mathrm{eV}$ is a multiphonon 
(a)

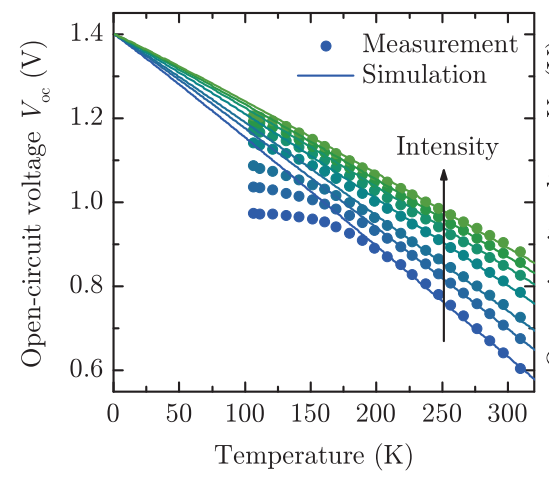

(b)

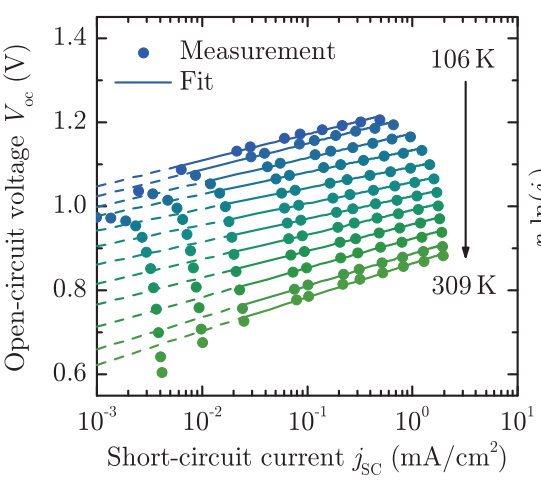

(c)

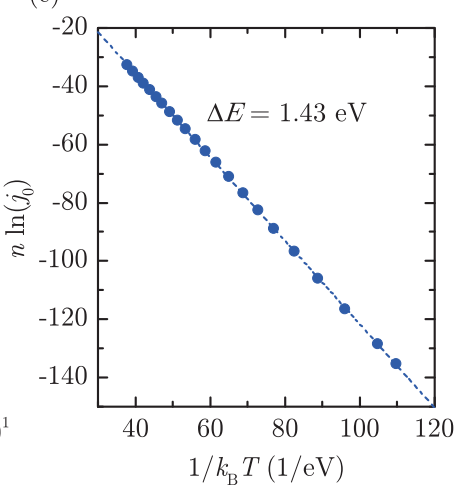

FIG. 5. (Color online) (a) Temperature dependence of $V_{\mathrm{OC}}$ measured for different illumination intensities. The solid lines show simulated behavior calculated with a modified SQ theory ${ }^{13}$ and $E_{\mathrm{CT}}=1.4 \mathrm{eV}$. (b) $V_{\mathrm{OC}}$ as a function of $j_{\mathrm{SC}}$ measured for different temperatures and illumination intensities. The straight lines are fits according to Eq. (3). (c) Temperature dependence of the dark saturation current evaluated by studying $n \ln \left(j_{0}\right)$ plotted vs $1 / k_{\mathrm{B}} T$ as given by the fit results extracted from (b). For clarity only a subset of intensities/temperatures is shown in (a) and (b).

absorption due to vibrational stretching modes of $\mathrm{C}-\mathrm{H}$ bonds, whereas the high-energy part $(>2.25 \mathrm{eV})$ is due to $\pi-\pi^{*}$ transitions (for details see Ref. 47). In $\mathrm{C}_{60}$ the increase of absorption already starts around $1.75 \mathrm{eV}$, in accordance with an optical gap commonly taken to be $1.9 \mathrm{eV}$. The spectrum of the mixed film of DIP: $\mathrm{C}_{60}$ is characterized by signatures of both materials: While at low energies the multiphonon absorption of DIP can be detected, the high-energy region is governed by the characteristic absorption of the fullerene, which implies that the main contribution to light absorption in a heterojunction device with comparable layer thickness of both materials comes from the fullerene. However, what clearly distinguishes the simple superposition of the single spectra from the measured spectrum of the blend film is the extra, broad absorption in the energy range between 1.3 and $1.7 \mathrm{eV}$ (hatched area), which cannot be found in any of the neat materials. This extra feature can be considered as a direct measure of the CT state as it presents the energetically lowest state to absorb light.

Figure 6(b) shows EL spectra of a PM-HJ DIP:C $\mathrm{C}_{60}$ solar cell (blend layer of $50 \mathrm{~nm}$ sandwiched between neat layers of 5-nm DIP and 10-nm $\mathrm{C}_{60}$ ) that was operated as an LED under varying forward bias, starting just above $V_{\mathrm{OC}}$. As in PDS, a coevaporated blend was chosen to increase the D/A interface, allowing for a larger signal from weak CT emission. The spectra are compared to those of single-layer devices with DIP and $\mathrm{C}_{60}$ (active layer of $100 \mathrm{~nm}$ ). The luminescence signals were corrected for the spectral sensitivity of the detector and normalized to their respective maximum values. The relative signal strengths are indicated by their magnification factors (given in brackets next to the respective curves). Due to the detection limit of the CCD camera the spectra are cut off at $1010 \mathrm{~nm}$.

The EL signal of a pure $\mathrm{C}_{60}$ device is extremely low and only detectable when applying a forward bias of $6 \mathrm{~V}$. One reason can be found in the symmetry-forbidden HOMO-LUMO transition of $\mathrm{C}_{60}:{ }^{48}$ Both the ground state $S_{0}$ and the first excited state $S_{1}$ exhibit even parity, which renders the transition between them dipole forbidden. However, vibronic coupling as well as disorder in the film may lead to a certain degree of mixed components of even and odd states, which can partially allow the violation of parity conservation. ${ }^{49}$ The results are comparable with literature values for the photoluminescence of $\mathrm{C}_{60}$ deposited on a quartz substrate, as found in Ref. 50: The authors attribute the main band at $E=1.71 \mathrm{eV}$ to the Frenkel exciton within one $\mathrm{C}_{60}$ molecule followed by its vibronic progression on the low-energy side.

The DIP single-layer device shows a pronounced EL signal already at moderate forward bias. The main EL peak positions are similar to those found by photoluminescence measurements, characterized by the $0-0$ transition at $E \approx 2.14 \mathrm{eV}$, which is followed by pronounced vibronic progression with peak energies of 1.98 and $1.80 \mathrm{eV} .^{47,51}$ The broad band around $1.7-1.6 \mathrm{eV}$ is assigned to an aggregate emission due to the high crystallinity of DIP. Apart from the spectra of the single-layer devices, Fig. 6(b) comprises the EL spectra of a PM-HJ solar cell. As expected, luminescence bands of both materials are identifiable in the wavelength range between 600 and $900 \mathrm{~nm}$ as the two materials form a phase-separated blend when they are evaporated simultaneously. ${ }^{25}$ However, EL offers the possibility to suppress the signals of the individual materials by reducing the applied voltage to a minimum, just above the open-circuit voltage. Thus, we observed a pronounced luminescence feature in the low-energy part of the spectrum (900-1000 nm), which becomes the dominant signature when the voltage is reduced to $V=1.1 \mathrm{~V}$. As there is no indication for light emission from any of the neat materials in this near-infrared spectral range, we attribute it to the transition from the interfacial CT state to the ground state. We note that the apparent shift of the peak maximum for increasing bias voltage reminds us of a Stark effect. However, the complex morphology of the blend film ${ }^{52}$ makes a detailed interpretation very difficult and is beyond the scope of this paper.

In a recent publication, Tvingstedt et al. report electroluminescence from several types of polymer-fullerene BHJ solar cells biased in the forward direction, deducing $E_{\mathrm{CT}}^{\mathrm{opt}}$ from the peak energy of the CT emission. ${ }^{53}$ This approach was refined by Vandewal et al.: ${ }^{20}$ Based on the framework of the 

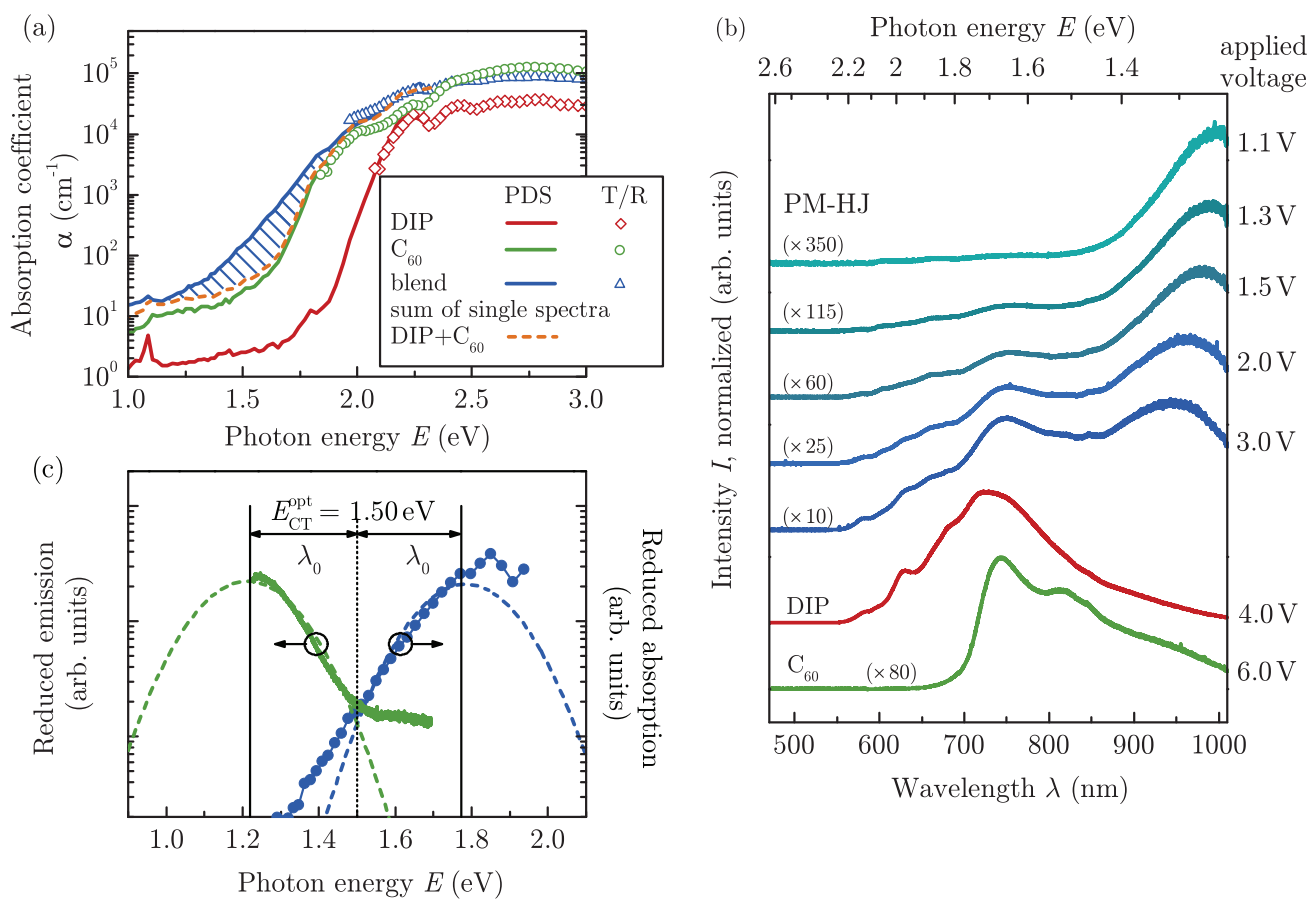

FIG. 6. (Color online) (a) Absorption spectrum for a blend of DIP:C ${ }_{60}$ [lines: photothermal deflection spectroscopy (PDS) ${ }^{13}$ open symbols: transmission/reflection measurement (T/R)]. For comparison, the single spectra of both materials as well as their sum are plotted to demonstrate the additional feature caused by the charge-transfer-state absorption. (b) Electroluminescence spectra of PM-HJ solar cells based on DIP: $\mathrm{C}_{60}$. The curves are obtained by applying a forward voltage of given magnitude. For better comparability all curves are normalized to their maximum values; however, the magnification factors are given in brackets to illustrate the strong variations in signal height. (c) Reduced absorption from PDS, blue circles, corresponding to the difference between the absorption spectrum of the blend and the sum of single absorption spectra) and emission (from EL, green straight line) for DIP: $\mathrm{C}_{60}$ measured at room temperature. The dashed curves are fits according to Eq. (11), using the same values for $E_{\mathrm{CT}}^{\mathrm{opt}}$ and $\lambda_{0}$ on both sides.

Marcus theory, the authors extracted the CT energy $E_{\mathrm{CT}}^{\mathrm{opt}}$ by analyzing the absorption and emission spectra of a series of polymer-PCBM blends. They fitted the reduced absorption $[\sigma(E) E]$ and emission $\left[I_{f}(E) / E\right]$ spectra of the CT manifold, respectively, by

$$
\left.\begin{array}{l}
\sigma(E) E \\
I_{\mathrm{f}}(E) E^{-1}
\end{array}\right\}=\frac{f_{\sigma, I_{\mathrm{f}}}}{\sqrt{4 \pi \lambda_{0} k_{\mathrm{B}} T}} \exp \left(\frac{-\left(E_{\mathrm{CT}}^{\mathrm{opt}} \pm \lambda_{0}-E\right)^{2}}{4 \lambda_{0} k_{\mathrm{B}} T}\right)
$$

As in Eq. (9), $\lambda_{0}$ denotes the reorganization energy (related to the Stokes shift). $f_{\sigma}$ and $f_{I_{\mathrm{f}}}$ are proportional to the square of the electronic coupling matrix element. The plus sign is used for the reduced absorption, the minus sign is used for the reduced emission.

The reduced absorption and emission spectra for the DIPand $\mathrm{C}_{60}$-based devices are shown in Fig. 6(c). By fitting both spectra with Eq. (11), a reorganization energy of $\lambda_{0} \approx 0.26 \mathrm{eV}$ as well as an energy of the CT state of $E_{\mathrm{CT}}^{\mathrm{opt}} \approx(1.50 \pm 0.06) \mathrm{eV}$ can be deduced. The reorganization energy $\lambda_{0}$ corresponds to nonradiative energy losses as a result of relaxation, and its magnitude is found to be similar to values determined for a variety of polymer-fullerene blends. ${ }^{20}$ Note, however, that a relatively large error of $0.06 \mathrm{eV}$ has to be assumed for $E_{\mathrm{CT}}^{\mathrm{opt}}$ since no clear absorption maximum is visible in the data, which reduces the reliability of the fit. Additional uncertainty is introduced by the fact that a mixed film has been used instead of a bilayer in order to increase the signal strengths. Furthermore, the potential temperature dependence of $E_{\mathrm{CT}}$ has not been investigated. If present, these effects are expected to be within $0.1 \mathrm{eV}^{20}$

\section{Discussion}

As a key outcome, the presented results convincingly show that different temperature-dependent electrical device characterizations and optical spectroscopies yield very similar values for the effective photovoltaic gap at the D/A interface in DIP/C 60 solar cells. The energy of the CT state $E_{\mathrm{CT}}^{\mathrm{opt}}=1.5 \mathrm{eV}$ obtained from PDS and EL spectra can be identified with the value $E_{\mathrm{CT}}=1.42 \mathrm{eV}$ extracted from the extrapolation of $V_{\mathrm{OC}}$ towards $0 \mathrm{~K}$. Both are in very good agreement with the activation energy $\Delta E=1.46 \mathrm{eV}$ extracted from the temperature dependence of the dark saturation current. In addition, we want to note that an interfacial energy gap $E_{\mathrm{DA}}=1.40 \pm 0.15 \mathrm{eV}$ at the $\mathrm{DIP} / \mathrm{C}_{60}$ heterojunction has recently been determined by means of photoelectron spectroscopy by Wilke et al. ${ }^{14}$ Together with this work, the correspondence between $E_{\mathrm{CT}}^{\mathrm{opt}}$, $E_{\mathrm{CT}}, \Delta E$, and $E_{\mathrm{DA}}$ is thus clearly demonstrated for the material system $\mathrm{DIP} / \mathrm{C}_{60}$. The a priori different energies are basically the same within an error of $0.1 \mathrm{eV}$. This fact qualifies the extrapolation of $V_{\mathrm{OC}}$ to $0 \mathrm{~K}$ and the analysis of the thermally activated $j_{0}$ as suitable methods to identify the intermolecular energy gap at the D/A interface of an organic heterojunction. These methods are especially helpful since they allow the 

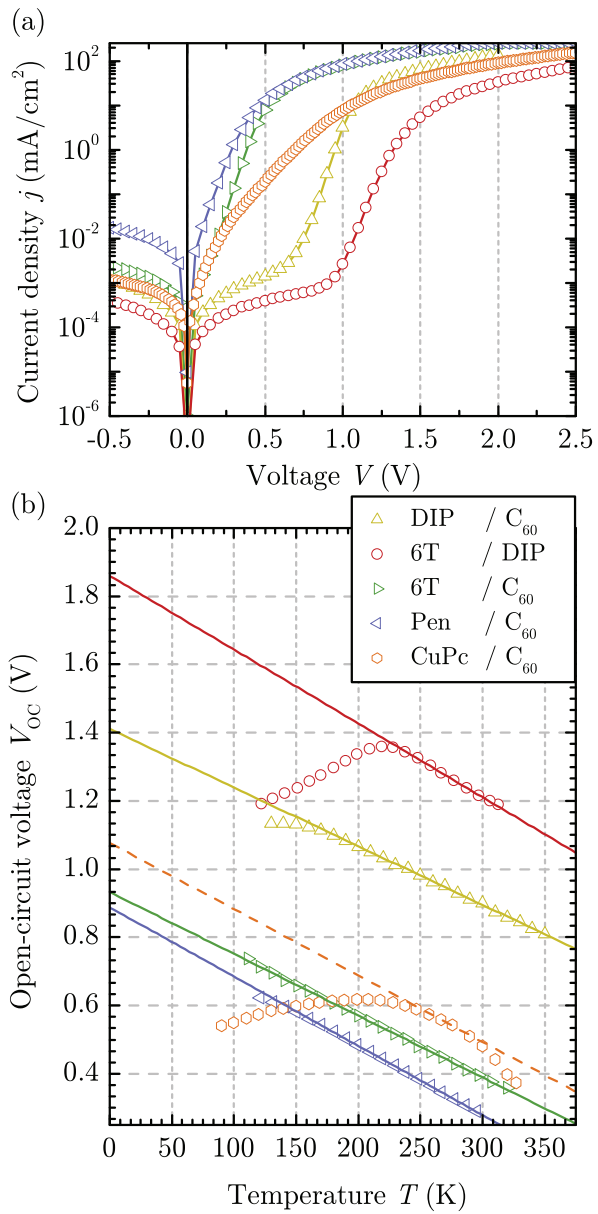

FIG. 7. (Color online) (a) Dark $j-V$ characteristics of a series of PHJ solar cells employing different donor-acceptor combinations recorded at room temperature. The respective device structures and parameters are summarized in Table I. (b) Temperature dependence of $V_{\mathrm{OC}}$ of different $\mathrm{PHJ}$ solar cells. The extrapolation of the linear regime towards $T=0 \mathrm{~K}$ gives a measure of the energy of the charge-transfer state $E_{\mathrm{CT}}$.

examination of the buried interface in device geometry. We would like to stress that even though the methods presented in Figs. 3 and 5 eventually rely on the analysis of $j_{0}$, they have to be regarded as substantially different. In particular the cell is analyzed in the dark in the former case and under illumination in the latter one.

\section{B. Application to other molecular D/A systems}

\section{Temperature-dependent $j-V$ characteristics in the dark and under illumination}

After the clear demonstration that temperature-dependent electrical device characteristics yield direct access to the effective photovoltaic energy gap at the D/A interface, which sets the upper limit for $V_{\mathrm{OC}}$ in organic heterojunction photovoltaic cells, we now apply this approach to a series of molecular D/A combinations. An overview of their currentvoltage characteristics in the dark and at room temperature is given in Fig. 7(a). Characteristic device parameters extracted from the fits according to the modified Shockley equation, i.e., $R_{\mathrm{S}} A, j_{0}$, and $n$, are given in Table I. An analysis of the temperature-dependent measurements was done for each material combination following the same procedure as exemplarily shown above for the DIP/ $\mathrm{C}_{60} \mathrm{PHJ}$ cell. The activation energy $\Delta E$ extracted from the temperature dependence of $j_{0}$ according to Eq. (2) is also included in Table I.

The characteristic solar-cell parameters under illumination, such as $V_{\mathrm{OC}}, j_{\mathrm{SC}}$, and FF, were determined for each cell at room temperature at illumination intensities of about $0.2-0.4$ sun (see Table I). Remarkably, there is a strong variation of $V_{\mathrm{OC}}$ between $\sim 0.3 \mathrm{~V}$ for Pen/ $\mathrm{C}_{60}$ and $1.24 \mathrm{~V}$ for 6T/DIP, which cannot be caused by the slight variation of $j_{\mathrm{SC}}$. As in the case of DIP/ $\mathrm{C}_{60}$, temperature-dependent $V_{\mathrm{OC}}$ measurements were extrapolated linearly towards $T=0 \mathrm{~K}$, yielding $E_{\mathrm{CT}}$. Figure 7(b) depicts the measured data for the different material systems (symbols) recorded in a temperature range of $110 \mathrm{~K}<$ $T<320 \mathrm{~K}$. The linear fits are included as straight lines, and the values for $E_{\mathrm{CT}}$ are summarized in Table I. In the case of $\mathrm{CuPc} / \mathrm{C}_{60}$ the selection of the fit data obviously has a strong impact on the value extracted for $E_{\mathrm{CT}}$. However, the dashed line in Fig. 7(b) indicates a fit that is consistent with the literature from both direct spectroscopy ${ }^{54}$ and extrapolation of $V_{\mathrm{OC}}(T) .{ }^{15}$

The experimentally determined values for $E_{\mathrm{CT}}$ are compared to values of the intermolecular energy gap $E_{\mathrm{DA}}$ as found in the literature. The good correlation confirms the suitability of our approach. Furthermore, for most of the heterojunctions remarkably good agreement between $\Delta E$ (as extracted from the temperature behavior of $j_{0}$ ) and $E_{\mathrm{CT}}$ (as extracted from the linear extrapolation of $V_{\mathrm{OC}}$ versus $T$ ) is observed. The only exception is the material combination $\mathrm{CuPc} / \mathrm{C}_{60}$, which shows a large discrepancy between both energies: The activation energy $\Delta E$ of the $\mathrm{CuPc} / \mathrm{C}_{60}$ system is almost twice as high as $E_{\mathrm{CT}}$. We attribute this to a different recombination mechanism, as will be discussed below.

Note that while $P e n / C_{60}$ and $6 \mathrm{~T} / \mathrm{C}_{60}$ show a linear behavior over the entire temperature range, the other material systems deviate from linearity below a certain temperature. Comparable effects are reported in the literature and have been explained by energetic disorder ${ }^{55}$ and injection barriers. ${ }^{56}$ We attribute the observed saturation or even decrease of $V_{\mathrm{OC}}$ to injection barriers and insufficient carrier mobility at low temperatures. This manifests in a strongly increased series resistance $R_{\mathrm{S}}$ visible in a reduced dark injection current [cf. Fig. 3(a)], which is accompanied by a distinct $S$ kink of the light $j-V$ curve around $V_{\mathrm{OC}}$. Additionally, this leads to a strongly reduced fill factor. Finally, $j_{\mathrm{SC}}$ is decreased, which hints at severe recombination losses [cf. Fig. 4(a)]. As predicted by Eq. (3), $V_{\mathrm{OC}}$ depends on $j_{\mathrm{SC}}$, representing the photocurrent. In the thermodynamic limit, which forms the basis for the linear extrapolation to $T=0 \mathrm{~K}$, however, the photocurrent solely depends on the exciton generation rate and is thus a constant value. By replacing the ideal photocurrent with the measured short-circuit current, additional recombination losses caused by $R_{\mathrm{S}}$ are included. This directly yields the sublinear dependence of $V_{\mathrm{OC}}$ observed at low temperatures.

Overall, the extrapolation of the temperature-dependent $V_{\mathrm{OC}}$ seems to represent a reliable method to determine $E_{\mathrm{CT}}$ not only for $\mathrm{DIP} / \mathrm{C}_{60}$ but also for a whole range of material combinations. This indicates a more general suitability of this method. 
TABLE I. Characteristic parameters for a series of PHJ photovoltaic devices with different donor-acceptor materials. All cells have a similar device structure of ITO/PEDOT $\approx 30 \mathrm{~nm}) / \mathrm{donor} /$ acceptor/BCP $(5 \mathrm{~nm}) / \mathrm{Al}$, with donor and acceptor layer thicknesses (in $\mathrm{nm})$ as given below. Quantities extracted from illuminated $j-V$ characteristics at room temperature are open-circuit voltage $V_{\mathrm{OC}}$, short-circuit current density $j_{\mathrm{sc}}$, and fill factor. Light illumination inside the cryostat was realized with a white LED with an estimated intensity $I_{\mathrm{L}}$. $E_{\mathrm{CT}}$ denotes the energy of the charge-transfer state as extracted from a linear extrapolation of the temperature-dependent $V_{\mathrm{OC}}$ towards $T=0 \mathrm{~K}$. The comparatively small differences in $I_{\mathrm{L}}$ [cf. Fig. 5(a)] are considered negligible for the values of $E_{\mathrm{CT}}$. Quantities extracted from dark $j$ - $V$ characteristics at room temperature are specific series resistance $R_{\mathrm{S}} A$, dark saturation current density $j_{0}$, and ideality factor $n$. $\Delta E$ denotes the activation energy as deduced from the temperature dependence of the dark saturation current. With these parameters $j_{00}$ was derived from Eq. (2). For comparison, the intermolecular energy gap $E_{\mathrm{DA}}=\left|E_{\mathrm{HOMO}}^{\text {donor }}-E_{\mathrm{LUMO}}^{\text {acceptor }}\right|$ as taken from literature values is listed.

\begin{tabular}{|c|c|c|c|c|c|c|c|c|c|c|c|}
\hline \multirow[b]{2}{*}{$\mathrm{D} / \mathrm{A}$} & \multicolumn{5}{|c|}{ Illuminated $j-V$ characteristics } & \multicolumn{5}{|c|}{ Dark $j$ - $V$ characteristics } & \multirow{2}{*}{$\begin{array}{c}\text { Literature } \\
E_{\mathrm{DA}} \\
(\mathrm{eV})\end{array}$} \\
\hline & $\begin{array}{l}V_{\mathrm{OC}} \\
(\mathrm{V})\end{array}$ & $\begin{array}{c}j_{\mathrm{SC}} \\
\left(\mathrm{mA} / \mathrm{cm}^{2}\right)\end{array}$ & $\begin{array}{l}\mathrm{FF} \\
(\%)\end{array}$ & $\begin{array}{c}I_{\mathrm{L}} \\
\left(\mathrm{mW} / \mathrm{cm}^{2}\right)\end{array}$ & $\begin{array}{l}E_{\mathrm{CT}} \\
(\mathrm{eV})\end{array}$ & $\begin{array}{c}R_{\mathrm{S}} A \\
\left(\Omega \mathrm{cm}^{2}\right)\end{array}$ & $\begin{array}{c}j_{0} \\
\left(\mathrm{~mA} / \mathrm{cm}^{2}\right)\end{array}$ & $n$ & $\begin{array}{c}\Delta E \\
(\mathrm{eV})\end{array}$ & $\begin{array}{c}j_{00} \\
\left(\mathrm{~mA} / \mathrm{cm}^{2}\right)\end{array}$ & \\
\hline $\operatorname{DIP}(50) / \mathrm{C}_{60}(80)$ & 0.91 & -1.3 & 63.6 & 18 & 1.42 & 5 & $1.0 \times 10^{-10}$ & 1.6 & 1.46 & $2.13 \times 10^{5}$ & 1.4 (Ref. 14) \\
\hline 6T(60)/DIP(60) & 1.24 & -1.3 & 42.5 & 37 & 1.86 & 14 & $8.0 \times 10^{-11}$ & 2.3 & 2.03 & $5.37 \times 10^{4}$ & 1.8 (Ref. 14) \\
\hline $6 \mathrm{~T}(40) / \mathrm{C}_{60}(80)$ & 0.41 & -2.3 & 60.0 & 32 & 0.93 & 6 & $7.1 \times 10^{-5}$ & 1.7 & 0.94 & $1.38 \times 10^{5}$ & 0.95 (Ref. 14) \\
\hline $\operatorname{Pen}(50) / \mathrm{C}_{60}(80)$ & 0.29 & -3.1 & 48.3 & 36 & 0.89 & 5 & $6.0 \times 10^{-3}$ & 2.1 & 0.98 & $4.15 \times 10^{5}$ & 1.05 (Refs. 57 and 58) \\
\hline $\mathrm{CuPc}(25) / \mathrm{C}_{60}(50)$ & 0.51 & -1.9 & 34.0 & 28 & 1.08 & 8 & $2.0 \times 10^{-3}$ & 4.9 & 1.98 & $1.23 \times 10^{4}$ & $1.0($ Ref. 31$)$ \\
\hline
\end{tabular}

\section{Energy losses from the CT state to open-circuit voltage}

Figure 8 summarizes the overall result of the present studies by showing the relation between $V_{\mathrm{OC}}$ at room temperature and $E_{\mathrm{CT}}$ of the different devices. Here, an excellent correlation is found for all of the material systems, clearly affirming that the open-circuit voltage is determined by the energy of the CT state, as predicted by Eq. (8). By applying a best fit to the data the linear dependence can be described by

$$
V_{\mathrm{OC}}=E_{\mathrm{CT}} / q-0.55 \mathrm{~V} .
$$

In this context, it has to be mentioned that the $V_{\mathrm{OC}}$ loss $\Delta V_{\mathrm{OC}}$ derived from experimental dark $j$ - $V$ curves by $q \Delta V_{\mathrm{OC}}=$ $n k_{\mathrm{B}} T \ln \left(j_{00} / j_{\mathrm{SC}}\right)[\mathrm{cf} . \mathrm{Eq}$. (4)] is a result of both radiative and nonradiative recombination currents. Separation of the two components can be achieved by simulation if the absorption strength of the CT state $\alpha_{\mathrm{CT}}$ is known. For a DIP/C $60 \mathrm{PHJ}$

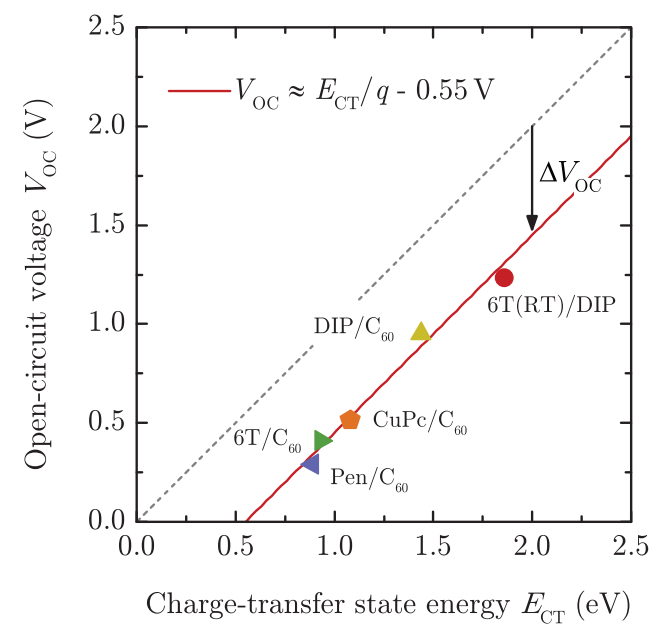

FIG. 8. (Color online) Relation between $V_{\mathrm{OC}}$ and $E_{\mathrm{CT}}$ (as extracted from the linear extrapolation of $V_{\mathrm{OC}}$ towards $0 \mathrm{~K}$ ) for the different studied photovoltaic devices as listed in Table I. Their linear dependence for all material combinations is confirmed by a fit according to $V_{\mathrm{OC}}=E_{\mathrm{CT}} / q-0.55 \mathrm{~V}$. The dashed line marks a one-to-one correspondence. cell this has been done by Gruber et al.; at room temperature $\Delta V_{\mathrm{OC}}^{(\mathrm{rad})} \approx 0.1 \mathrm{~V}$ and $\Delta V_{\mathrm{OC}}^{(\text {nonrad })} \approx 0.4 \mathrm{~V}$ were found. ${ }^{13}$

This indicates that in all of the investigated molecular D/A cells there is an energy loss of roughly half a volt between the energy of the CT state and $V_{\mathrm{OC}}$ at room temperature and that the dominant fraction is nonradiative losses.

\section{Discussion}

For most of the investigated heterojunctions we could observe remarkably good agreement between $\Delta E$ (as extracted from the temperature behavior of $j_{0}$ ) and $E_{\mathrm{CT}}$ (as extracted from the linear extrapolation of $V_{\mathrm{OC}}$ versus $T$ ). The direct correlation between $\Delta E$ and $E_{\mathrm{CT}}$ indicates with great confidence that the activation energy for the dark saturation current $\Delta E$ corresponds in a one-to-one manner to the CT energy. Both quantities represent the effective energy gap for an organic heterojunction solar cell. The only material system which shows deviations from this general rule is the $\mathrm{CuPc} / \mathrm{C}_{60}$ heterojunction. This deviation is already indicated by the shape of the dark $j-V$ curves: As can be seen from Fig. 7(a), the current in the diffusion-dominated voltage regime is characterized by two differently steep slopes, a feature which becomes less pronounced at low temperatures. In literature, this double-exponential $j-V$ characteristic observed for $\mathrm{CuPc} / \mathrm{C}_{60}$ has been addressed by the introduction of two independent diode ideality factors which consider the recombination of free acceptor electrons with trapped donor holes and vice versa. ${ }^{24}$ Another peculiarity of $\mathrm{CuPc} / \mathrm{C}_{60}$ is that $\mathrm{CuPc}$ shows very fast intersystem crossing and that its $T_{1}$ triplet state lies energetically close to the donor-acceptor CT state. ${ }^{54,59,60}$ The exceptional importance of triplets at this interface might possibly lead to a more complex recombination mechanism compared to other systems. In any case, this observation suggests that $\mathrm{CuPc} / \mathrm{C}_{60}$ is not the material combination of choice to investigate a correlation between $\Delta E$ and $E_{\mathrm{CT}}$. The data might lead to the assumption that $\Delta E$ corresponds to twice the effective band gap. ${ }^{21,23,61}$ This is, however, not true for a broad range of other material systems and needs to be carefully reviewed. 
Concerning the band-gap-voltage offset, i.e., the difference between the effective gap at the heterojunction (e.g., $E_{\mathrm{CT}} / q$ ) and the $V_{\mathrm{OC}}$ measured at room temperature, Fig. 8 clearly shows that about $0.5-0.6 \mathrm{~V}$ is lost for each investigated heterojunction. These losses are caused by recombination, both radiative and nonradiative. Both parts can be summarized in one experimentally accessible quantity $\Delta V_{\mathrm{OC}} \propto \ln \left(j_{00} / j_{\mathrm{SC}}\right)$. $j_{00}$ is generally considered to be a measure of the intermolecular coupling between the donor and acceptor molecules, where low $j_{00}$ corresponds to weak coupling, a property which can be modified by varying the strength of the $\pi$ interaction. ${ }^{23,29,61,62}$ Consequently, the experimentally apparent independence of the absolute $V_{\mathrm{OC}}$ loss on the material system becomes evident by considering these coupling constants (cf. Table I). Even though $j_{00}$ varies by roughly one order of magnitude, the value enters logarithmically into $\Delta V_{\mathrm{OC}}$. This means that the electronic coupling is virtually identical for all investigated heterojunctions, independent of the effective photovoltaic energy gap. This results in very similar absolute losses. Even though a high short-circuit current will also favor a high $V_{\mathrm{OC}}$, direct comparison between the values found for $j_{00}$ and $j_{\text {SC }}$ shows that the coupling constant dominates the impact on $V_{\mathrm{OC}}$ by far.

These findings emphasize that in order to achieve the highest possible $V_{\mathrm{OC}}$ two fundamental requirements have to be met by the material system: (1) The effective gap (be it $\Delta E$ or $E_{\mathrm{CT}}$ ) has to be as large as possible in order to enable a large chemical potential of the charge-carrier pairs. (2) Recombination has to be suppressed to a minimum in order to maintain the chemical potential. The second requirement crucially depends on minimizing the coupling constant $j_{00}$ while at the same time keeping $j_{\text {SC }}$ high. Note that considering the mechanisms of charge generation in excitonic solar cells, weak electronic coupling at the D/A interface and large $j_{\mathrm{SC}}$ have to be regarded as potentially concurring aims. However, the predominant recombination losses are nonradiative and thus avoidable. ${ }^{13,43}$

The fact that $\Delta V_{\mathrm{OC}}$ is identical for all systems implies that the relative loss with respect to the particular effective photovoltaic gap is larger for material combinations with small intermolecular gaps. This is shown in Fig. 9. The measured relative $V_{\mathrm{OC}}$ loss with respect to $E_{\mathrm{CT}} / q$ derived from solar-cell characteristics under illumination (i.e., $1-q V_{\mathrm{OC}} / E_{\mathrm{CT}}$ ) is plotted vs the predicted relative losses derived from dark $j-V$ characteristics using Eq. (4) [i.e., $\left.\left(n k_{B} T / \Delta E\right) \ln \left(j_{00} / j_{\mathrm{SC}}\right)\right]$. The almost-perfect one-toone match of predicted and measured values shows that $V_{\mathrm{OC}}$ losses observed for the illuminated solar cell emerge already from the dark behavior of the diode, presumably from the strength of the donor-acceptor electronic coupling. This again underlines the fact that both techniques for the determination of the effective photovoltaic energy gap are fully equivalent.

In passing, we want to note that, interestingly, even inorganic single-junction solar cells exhibit band-gap-voltage offsets between 0.4 and $0.6 \mathrm{eV}$, more or less independent of the absolute magnitude of the semiconductor band gap. ${ }^{63}$ This may be taken as a further confirmation that both organic and inorganic heterojunctions obey basically the same physics.

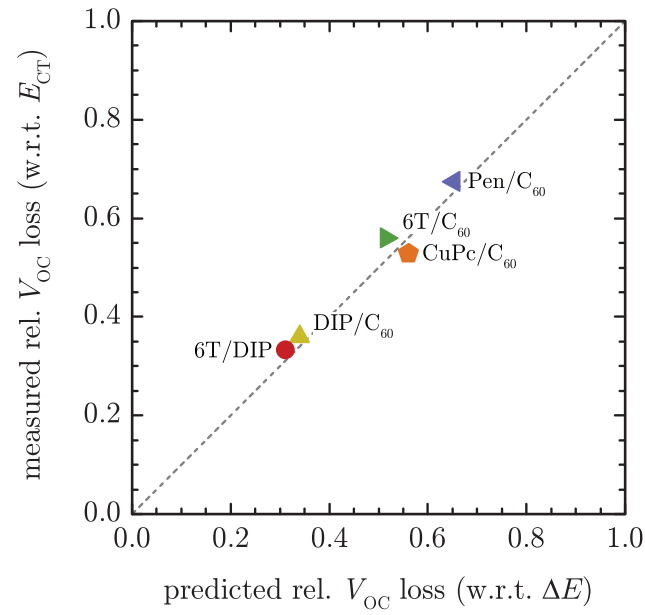

FIG. 9. (Color online) Relative $V_{\mathrm{OC}}$ loss of the different studied photovoltaic devices as listed in Table I, measured under illumination vs the predicted relative loss using parameters from dark characteristics and Eq. (4). Measured values are normalized by $E_{\mathrm{CT}}$; predicted values are normalized by $\Delta E$ (extracted from light and dark characteristics, respectively). The dashed line marks a one-to-one correspondence.

\section{CONCLUSIONS}

In this paper the effective photovoltaic gap acting as the upper limit of the open-circuit voltage of organic molecular heterojunction solar cells has been analyzed, and energy losses have been identified. For this purpose the quantities $\Delta E, E_{\mathrm{CT}}$, and $E_{\mathrm{DA}}$ typically used interchangeably in the literature have a priori been considered to be independent. In this scope, $\Delta E$ denotes the activation energy of the dark saturation current and can be regarded as the minimum energy required for charge generation. $E_{\mathrm{CT}}$ denotes the energy of the lowest radiative transition and is thus dominated by recombination. $E_{\mathrm{DA}}$ is the D/A intermolecular gap as determined by photoemission spectroscopy. These quantities were then shown to have equal values, indeed rendering them interchangeable.

In the first part of this work a thorough investigation of the CT energies of planar heterojunction $\mathrm{DIP} / \mathrm{C}_{60}$ cells was carried out. $\Delta E$ and $E_{\mathrm{CT}}$ were determined by two complementary methods each. While $\Delta E$ was extracted from the temperature dependence of the dark saturation current in both cases, $j_{0}$ itself was determined by distinctly different experiments. In the first case the dark injection current under forward bias was evaluated; in the second case the intensity dependence of $V_{\mathrm{OC}}$ was analyzed. Despite completely different solar-cell conditions, almost-identical results were extracted. $E_{\mathrm{CT}}$ was extracted from the temperature dependence of $V_{\mathrm{OC}}$ by extrapolation to $0 \mathrm{~K}$. This value was compared to direct spectroscopic detection of the CT state by combined analysis of emission and absorption. Again, a close match of both values was obtained. By comparison of $\Delta E, E_{\mathrm{CT}}$, and $E_{\mathrm{DA}}$ from the literature (determined by photoemission and inverse photoemission spectroscopy $)^{14}$ all three values could clearly be identified to be identical for the DIP/C 60 system. This shows that all four methods may reliably be applied to retrieve the effective energy gap at organic heterojunctions. 
In the second part the temperature dependences of the dark injection current and of $V_{\mathrm{OC}}$ were analyzed for a series of material systems. Good agreement of $\Delta E$ and $E_{\mathrm{CT}}$ was found for all systems except $\mathrm{CuPc} / \mathrm{C}_{60}$, where recombination via trap states or triplets is assumed to play a major role. ${ }^{24,59}$ The general rule well established for bulkheterojunction solar cells, that $V_{\mathrm{OC}}$ typically is $0.5-0.6 \mathrm{~V}$ below the effective photovoltaic gap, could clearly be verified for molecular planar heterojunction devices. Furthermore, we attribute this apparent generality to similar donor-acceptor electronic coupling strength, as indicated by relatively small variations of $j_{00}$. Additionally, we could show that the relative $V_{\mathrm{OC}}$ loss observed under illumination can already be predicted from the dark characteristics of the solar cell. The overall good agreement of values macroscopically observed and calculated from microscopic quantities shows that the temperature-dependent analysis of solar-cell characteristics provides a simple and convenient tool to probe the effective energy gap at the donor-acceptor interface directly in device geometry.

\section{ACKNOWLEDGMENTS}

This work was supported by the German Research Foundation (DFG) within the priority program SPP 1355 ("Elementary Processes of Organic Photovoltaics") and by the Bavarian State Ministry of Science, Research and the Arts within the collaborative research network "Solar Technologies go Hybrid.'U.H. and S.G. acknowledge the Bavarian Research Foundation for financial support. H.J.K. and S.K. acknowledge support by the DFG via the Emmy Noether Program (KR3790/2-1). The Elite Network of Bavaria within the International Graduate School: Materials Science of Complex Interfaces is gratefully acknowledged by M.G. *wolfgang.bruetting@physik.uni-augsburg.de

${ }^{1}$ http://www.nrel.gov/ncpv/.

${ }^{2}$ http://www.heliatek.com.

${ }^{3}$ R. A. J. Jansen and J. Nelson, Adv. Mater. 25, 1847 (2013).

${ }^{4}$ W. Shockley and H. J. Queisser, J. Appl. Phys. 32, 510 (1961).

${ }^{5}$ B. A. Gregg and M. C. Hanna, J. Appl. Phys. 93, 3605 (2003).

${ }^{6}$ D. Veldman, S. C. J. Meskers, and R. A. J. Janssen, Adv. Funct. Mater. 19, 1939 (2009).

${ }^{7}$ T. M. Clarke and J. R. Durrant, Chem. Rev. 110, 6736 (2010).

${ }^{8}$ C. Deibel, T. Strobel, and V. Dyakonov, Adv. Mater. 22, 4097 (2010).

${ }^{9} \mathrm{~K}$. Vandewal, K. Tvingstedt, and O. Inganäs, in Quantum Efficiency in Complex Systems, Part II: From Molecular Aggregates to Organic Solar Cells, edited by M. T. Uli Würfel and E. R. Weber, Semiconductors and Semimetals Vol. 85 (Elsevier, Amsterdam, 2011), pp. 261-295.

${ }^{10}$ T. Kirchartz, J. Mattheis, and U. Rau, Phys. Rev. B 78, 235320 (2008).

${ }^{11}$ N. C. Giebink, G. P. Wiederrecht, M. R. Wasielewski, and S. R. Forrest, Phys. Rev. B 83, 195326 (2011).

${ }^{12}$ L. J. A. Koster, S. E. Shaheen, and J. C. Hummelen, Adv. Energy Mater. 2, 1246 (2012).

${ }^{13}$ M. Gruber, J. Wagner, K. Klein, U. Hörmann, A. Opitz, M. Stutzmann, and W. Brütting, Adv. Energy Mater. 2, 1100 (2012).

${ }^{14}$ A. Wilke, J. Endres, U. Hörmann, J. Niederhausen, R. Schlesinger, J. Frisch, P. Amsalem, J. Wagner, M. Gruber, A. Opitz, A. Vollmer, W. Brütting, A. Kahn, and N. Koch, Appl. Phys. Lett. 101, 233301 (2012).

${ }^{15}$ J. Widmer, M. Tietze, K. Leo, and M. Riede, Adv. Funct. Mater. (to be published).

${ }^{16}$ M. C. Scharber, D. Mühlbacher, M. Koppe, P. Denk, C. Waldauf, A. J. Heeger, and C. J. Brabec, Adv. Mater. 18, 789 (2006).

${ }^{17}$ R. R. Lunt, T. P. Osedach, P. R. Brown, J. A. Rowehl, and V. Bulović, Adv. Mater. 23, 5712 (2011).

${ }^{18}$ K. Vandewal, A. Gadisa, W. D. Oosterbaan, S. Bertho, F. Banishoeib, I. Van Severen, L. Lutsen, T. J. Cleij, D. Vanderzande, and J. V. Manca, Adv. Funct. Mater. 18, 2064 (2008).
${ }^{19}$ K. Vandewal, K. Tvingstedt, A. Gadisa, O. Inganäs, and J. V. Manca, Nat. Mater. 8, 904 (2009).

${ }^{20} \mathrm{~K}$. Vandewal, K. Tvingstedt, A. Gadisa, O. Inganäs, and J. V. Manca, Phys. Rev. B 81, 125204 (2010).

${ }^{21}$ B. P. Rand, D. P. Burk, and S. R. Forrest, Phys. Rev. B 75, 115327 (2007).

${ }^{22}$ W. J. Potscavage, A. Sharma, and B. Kippelen, Acc. Chem. Res. 42, 1758 (2009).

${ }^{23}$ M. D. Perez, C. Borek, S. R. Forrest, and M. E. Thompson, J. Am. Chem. Soc. 131, 9281 (2009).

${ }^{24}$ N. C. Giebink, G. P. Wiederrecht, M. R. Wasielewski, and S. R. Forrest, Phys. Rev. B 82, 155305 (2010).

${ }^{25}$ J. Wagner, M. Gruber, A. Hinderhofer, A. Wilke, B. Bröker, J. Frisch, P. Amsalem, A. Vollmer, A. Opitz, N. Koch, F. Schreiber, and W. Brütting, Adv. Funct. Mater. 20, 4295 (2010).

${ }^{26}$ S. Sze, Physics of Semiconductor Devices (Wiley, New York, 1982).

${ }^{27}$ J. Nelson, The Physics of Solar Cells (Imperial College Press, London, 2003).

${ }^{28}$ A. Fahrenbruch and J. Aranovich, in Solar Energy Conversion, edited by B. Seraphin,Topics in Applied Physics Vol. 31 (Springer, Berlin, 1979), pp. 257-326.

${ }^{29}$ S. Yamamoto, A. Orimo, H. Ohkita, H. Benten, and S. Ito, Adv. Energy Mater. 2, 229 (2012).

${ }^{30}$ U. Rau, Phys. Rev. B 76, 085303 (2007).

${ }^{31}$ A. Wilke, T. Mizokuro, R.-P. Blum, J. P. Rabe, and N. Koch, IEEE J. Sel. Top. Quantum Electron. 16, 1732 (2010).

${ }^{32}$ S. Duhm, I. Salzmann, G. Heimel, M. Oehzelt, A. Haase, R. L. Johnson, J. P. Rabe, and N. Koch, Appl. Phys. Lett. 94, 033304 (2009).

${ }^{33}$ I. Hill, A. Kahn, Z. Soos, and R. Pascal, Chem. Phys. Lett. 327, 181 (2000).

${ }^{34}$ A. Kahn, N. Koch, and W. Gao, J. Polym. Sci., Part B 41, 2529 (2003).

${ }^{35}$ P. I. Djurovich, E. I. Mayo, S. R. Forrest, and M. E. Thompson, Org. Electron. 10, 515 (2009).

${ }^{36}$ J. Wagner, M. Gruber, A. Wilke, Y. Tanaka, K. Topczak, A. Steindamm, U. Hörmann, A. Opitz, Y. Nakayama, H. Ishii, 
J. Pflaum, N. Koch, and W. Brütting, J. Appl. Phys. 111, 054509 (2012).

${ }^{37}$ D. Kurrle and J. Pflaum, Appl. Phys. Lett. 92, 133306 (2008).

${ }^{38}$ M. Horlet, M. Kraus, W. Brütting, and A. Opitz, Appl. Phys. Lett 98, 233304 (2011).

${ }^{39}$ S. Yoo, B. Domercq, and B. Kippelen, J. Appl. Phys. 97, 103706 (2005).

${ }^{40}$ J. D. Servaites, S. Yeganeh, T. J. Marks, and M. A. Ratner, Adv. Funct. Mater. 20, 97 (2010).

${ }^{41}$ G. A. H. Wetzelaer, L. J. A. Koster, and P. W. M. Blom, Phys. Rev. Lett. 107, 066605 (2011).

${ }^{42}$ V. D. Mihailetchi, P. W. M. Blom, J. C. Hummelen, and M. T. Rispens, J. Appl. Phys. 94, 6849 (2003).

${ }^{43} \mathrm{~K}$. Vandewal, K. Tvingstedt, J. Manca, and O. Inganäs, IEEE J. Sel. Top. Quantum Electron. 16, 1676 (2010).

${ }^{44}$ M. A. Green, Prog. Photovoltaics 11, 333 (2003).

${ }^{45} \mathrm{P}$. Würfel, Physics of Solar Cells: From Basic Principles to Advanced Concepts (Wiley-VCH, Weinheim, 2009).

${ }^{46}$ K. S. Nalwa, H. K. Kodali, B. Ganapathysubramanian, and S. Chaudhary, Appl. Phys. Lett. 99, 263301 (2011).

${ }^{47}$ D. Zhang, A. Horneber, J. Mihaljevic, U. Heinemeyer, K. Braun, F. Schreiber, R. Scholz, and A. Meixner, J. Lumin. 131, 502 (2011). ${ }^{48}$ N. Sariciftci, Prog. Quantum Electron. 19, 131 (1995).

${ }^{49}$ M. Fox, Optische Eigenschaften von Festkörpern (Old enbourg Wissenschaftsverlag, Munich, Germany, 2012).

${ }^{50}$ V. Capozzi, G. Casamassima, G. Lorusso, A. Minafra, R. Piccolo, T. Trovato, and A. Valentini, Solid State Commun. 98, 853 (1996).
${ }^{51}$ M. Heilig, M. Domhan, and H. Port, J. Lumin. 110, 290 (2004).

${ }^{52}$ M. Gruber, M. Rawolle, J. Wagner, D. Magerl, U. Hörmann, J. Perlich, S. V. Roth, A. Opitz, F. Schreiber, P. Müller-Buschbaum, and W. Brütting, Adv. Energy Mater. 3, 1075 (2013).

${ }^{53}$ K. Tvingstedt, K. Vandewal, A. Gadisa, F. Zhang, J. Manca, and O. Inganäs, J. Am. Chem. Soc. 131, 11819 (2009).

${ }^{54}$ F. Piersimoni, D. Cheyns, K. Vandewal, J. V. Manca, and B. P. Rand, J. Phys. Chem. Lett. 3, 2064 (2012).

${ }^{55}$ A. Manor and E. A. Katz, Sol. Energy Mater. Sol. Cells 97, 132 (2012).

${ }^{56}$ D. Rauh, A. Wagenpfahl, C. Deibel, and V. Dyakonov, Appl. Phys. Lett. 98, 133301 (2011).

${ }^{57}$ I. Salzmann, S. Duhm, R. Opitz, R. L. Johnson, J. P. Rabe, and N. Koch, J. Appl. Phys. 104, 114518 (2008).

${ }^{58}$ J. C. Nolasco, A. Sánchez-Díaz, R. Cabré, J. Ferré-Borrull, L. F. Marsal, E. Palomares, and J. Pallarès, Appl. Phys. Lett. 97, 013305 (2010).

${ }^{59}$ G. J. Dutton and S. W. Robey, J. Phys. Chem. C 116, 19173 (2012).

${ }^{60}$ A. E. Jailaubekov, A. P. Willard, J. R. Tritsch, W.-L. Chan, N. Sai, R. Gearba, L. G. Kaake, K. J. Williams, K. Leung, P. J. Rossky, and X.-Y. Zhu, Nat. Mater. 12, 66 (2013).

${ }^{61}$ P. Erwin and M. E. Thompson, Appl. Phys. Lett. 98, 223305 (2011).

${ }^{62}$ C. W. Schlenker and M. E. Thompson, Chem. Commun. 47, 3702 (2011).

${ }^{63}$ R. R. King, D. Bhusari, A. Boca, D. Larrabee, X.-Q. Liu, C. M. Fetzer, D. C. Law, and N. H. Karam, Prog. Photovoltaics 19, 797 (2011). 